\title{
Suelos asociados a tres comunidades vegetales de pastoreo importantes en Patagonia Sur
}

\author{
Soils associated to three important grazing vegetal \\ communities in South Patagonia
}

\author{
Valle S. ${ }^{a}$, Radic S. ${ }^{b}$, Casanova M. ${ }^{* c}$ \\ ${ }^{a}$ Universidad Austral de Chile, Instituto de Ingeniería Agraria y Suelos, \\ Centro de Investigación en Suelos Volcánicos (CISVo); susanavalle@uach.cl \\ ${ }^{b}$ Universidad de Magallanes, Facultad de Ciencias; sergio.radic@umag.cl \\ ${ }^{c}$ Universidad de Chile, Departamento de Ingeniería y Suelos-Facultad de Ciencias Agronómicas; \\ mcasanov@uchile.cl
}

A R T I C L E IN F O

Article history:

Received 16.09.2015

Accepted 06.01.2016

Keywords:

Glacio-lacustrine soils

Tussock

Empetrum rubrum

Short Communication,

Special Edition: International

Year of Soils (IYS)

Soil Science

${ }^{*}$ Corresponding author:

Manuel Casanova

E-mail address:

mcasanov@uchile.cl
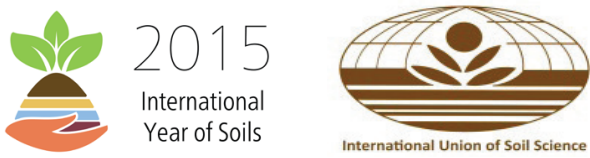

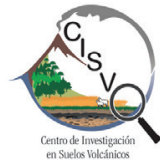

A B S T R A C T

South Patagonia (south of $53^{\circ} \mathrm{S}$ ) has long been famous for its rugged terrain, extremely oceanic climate and often hydromorphic vegetation and soil. Volcanism, tectonic and glacial processes are imprinting their actions along a territory, with vegetation-soil patterns that has been known since Darwin's time. Soil fertility and rainfalls seem to control the overall floristic gradient in a simple structure, often changed by anthropic disturbations. The goal of this study was to establish general relationships between three major plant communities (Empetrum rubrum or "murtilla", Festuca gracillima or "coirón", and hydromorphic Poas or "vegas") and the properties of fluvio-glacial mineral soils that support them. Soil under "murtilla" shows more constraints to plant growth, determined by a low $\mathrm{pH}$, high aluminum activity and low amounts of non acid cations.

\section{RESUMEN}

Patagonia Sur (al sur de $53^{\circ} \mathrm{S}$ ) se caracteriza por una geomorfología accidentada, un clima extremadamente oceánico y frecuente vegetación y suelos hidromórficos. Tanto el volcanismo, como los procesos tectónicos y glaciares han dejado sus huellas en el territorio, con patrones vegetación-suelo conocidos desde los primeros reconocimientos de Darwin. El gradiente de las precipitaciones y la fertilidad del suelo explican los cambios florísticos generales en una estructura simple, a menudo modificados por la acción del hombre. El objetivo de este trabajo fue establecer relaciones generales entre tres comunidades vegetales dominantes en la zona (murtillar de Empetrum rubrum, coironal de Festuca gracillima y vegas o mallines de pastos en condiciones hidromórficas) y las propiedades de los suelos de origen fluvioglacial que las sustentan. Los suelos bajo el murtillar muestran las mayores limitaciones para el crecimiento vegetal, determinadas por un bajo pH, una elevada actividad del ión aluminio y bajos niveles de cationes no ácidos.

Palabras clave: suelos glacio-lacustres, tussock, Empetrum rubrum.

\section{INTRODUCCIÓN}

La formación de patrones espaciales regulares de la vegetación se atribuye a la microtopografía del terreno (McGrath et al., 2012), a la variabilidad espacial de las propiedades del suelo (Maestre y Cortina, 2002) y a fenómenos globales como el cambio climático (Rietkerk et al., 2002; Dekker et al., 2010), entre otros.
En la Patagonia Chilena, se estima que cuatro componentes ambientales son de gran importancia para la composición, distribución, fisonomía y dinámica de la vegetación: la geomorfología, el clima, los suelos y las perturbaciones.

En Chile, las descripciones florísticas y fitosociológicas de la vegetación señalan agrupaciones asociativas con ciertos grados de artificialización, las que se 
inician a escalas variables (Pisano, 1973, 1974 y 1977; Rodríguez, 1986; Cruz y Lara, 1987). Para el extremo sur de Patagonia, SAG (2003, 2004a y 2004b) destaca al menos siete comunidades vegetales importantes, generalmente asociadas a suelos minerales: bosques, praderas, turba, mata, murtilla, coironal y vegas. Entre estas, las tres últimas representan más del $47 \%$ de la superficie de uso ganadero prospectada por el SAG.

Bajo el concepto de coironal se incluye a una comunidad vegetal donde dominan principalmente las especies Festuca gracillima o F. magellanica, una formación que ocupa un $24 \%$ del total de la superficie destinada a uso ganadero (SAG 2003, 2004a y 2004b). Se caracteriza por ser una formación herbácea de crecimiento cespitoso que considera, además de las festucas nativas, estipas y algunas poas. Este conjunto determina finalmente una formación de plantas que se desarrolla de un vigor variable, dependiendo de las características del sitio, especialmente de la disponibilidad hídrica. Además, se señala que entre las champas de coirón, crecen muchas dicotiledóneas y gramíneas bajas, fundamentalmente perennes con rizomas y estolones que en conjunto forman el cojín, recurso forrajero preferido por los ovinos (SAG, 2003).

La vega corresponde a una comunidad azonal (mallín) pratiforme higrófita de plantas herbáceas, con fisonomía de gramíneas cespitosas, asociadas en la estepa magallánica con depresiones del relieve u otras extensiones con subsuelos impermeables donde existe una condición hidromórfica permanente o estacional (Pisano, 1993; SAG, 2003). Su composición florística cambia de acuerdo a la disponibilidad de agua y la salinidad, dando origen a varios tipos de vega. Constituyen recursos de alta producción y valor forrajero, de gran importancia como complemento para la ganadería de la zona.

En el murtillar domina principalmente Empetrum rubrum Vahl ex Willd (murtilla), un arbusto postrado dioico que crece en Patagonia Chilena y Argentina. Se observa en planicies con buen drenaje, y en pendientes expuestas a vientos extremos, donde la nieve de invier- no puede prolongarse por varios meses (Roig, 1998). En Tierra del Fuego, E. rubrum forma importantes comunidades sub-arbustivas de la estepa magallánica, asociada a suelos de textura gruesa, pobres en nutrientes y ácidos (Collantes et al., 1989; 1999). Debido a la distribución geográfica de esta especie, se asume que tuvo un rol pionero en ocupar esas áreas, desde las cuales el hielo fue desapareciendo a finales de la última glaciación (Roig, 1998). Varios autores (Collantes et al., 1999; Anchorena et al., 2001) señalan que la crianza intensiva de ovinos en la estepa magallánica pudo propiciar el aumento en la degradación de las praderas, dando paso al dominio de E. rubrum en variadas proporciones, y otros arbustos postrados acidofílicos de poco valor forrajero.

Tanto Patagonia como Tierra del Fuego muestran un registro amplio y variado de la evolución costera, que comprende complejas relaciones entre depósitos glaciales, alturas interglaciares y diferentes tendencias de levantamiento (Isla y Bujalesky, 2008). Así, dentro de este complejo tectónico (placas Scotia-AntárticaSudamericana), volcánico y fluvio-glaciar, el clima ha ejercido gran influencia en la formación de los suelos a través de las precipitaciones, temperaturas y el viento.

Se han realizado numerosos estudios agrológicos sistemáticos en el extremo sur de Patagonia (Cuadro 1). Zaixso y Boraso (2015) señalan que el hidromorfismo permanente de la zona expone a los sitios a una mayor presión de pastoreo y pisoteo, transformándolos en áreas muy degradadas. La sensibilidad de estos ambientes a la erosión es alta por la pobre estructura de los suelos y la falta de fragmentos gruesos. En particular CIREN (2010), señala que en la Región de Magallanes y Antártica Chilena existe una superficie actual de suelos erosionados (erosión ligera, moderada, severa y muy severa) en un $28,5 \%$ de la superficie regional, parte de la cual ha sido afectada durante milenios por procesos de erosión geológica.

Por otra parte, las explotaciones petroleras y mineras, cuando no se ajustan a las normas de protección ambiental, desencadenan procesos erosivos que im-

Cuadro 1. Estudios agrológicos sistemáticos en Patagonia Sur.

Table 1. Systematic soil surveys performed in South Patagonia.

\begin{tabular}{ccccc}
\hline Escala & Unidad cartográfica & Coordenadas & Superficie(ha) & Referencia \\
\hline $1: 500.000$ & Gran Grupo & $51^{\circ}$ a $54^{\circ} \mathrm{S} / 68^{\circ}$ a $73^{\circ} \mathrm{W}$ & 2.555 .000 & Díaz et al. (1959-1960) \\
$1: 50.000$ & Asociaciones & $52^{\circ}$ a $53,5^{\circ} \mathrm{S} / 70^{\circ}$ a $71^{\circ} \mathrm{W}$ & 946.262 & IREN (1968) \\
$\sim 1: 250.000$ & Familias & $52^{\circ}$ a $56^{\circ} \mathrm{S} / 65^{\circ}$ a $69^{\circ} \mathrm{W}$ & $\sim 300.000$ & Frederiksen (1988) \\
$1: 50.000$ & Fases de Series & $48^{\circ}$ a $54^{\circ} \mathrm{S} / 68^{\circ}$ a $72^{\circ} \mathrm{W}$ & 100.000 & CNR (1997) \\
\hline
\end{tabular}


plican un empobrecimiento del suelo y una reducción de la riqueza florística (Cibils y Borelli, 2005; Paruelo et al., 2006). La influencia del hombre sobre estos ambientes se manifiesta además por la introducción de plantas exóticas, que inicialmente se instalan solamente en terrenos removidos, pero pueden llegar a desplazar exitosamente a las comunidades naturales y con el tiempo provocar alteraciones importantes en el ecosistema (Zaixso y Boraso, 2015). Además, el modo de pastoreo de los ovinos en las estepas magallánicas, ha transformado muchas comunidades de gramíneas en praderas degradadas (Collantes et al., 1999; Ramírez et al., 2012).

Abundante literatura existe acerca de la asociación suelo-bosque (Gerding y Thiers, 2002; Romanyá et al., 2005), así como también de caracterización de suelos orgánicos (Filipová et al., 2010; Loisel y Yu, 2013). No obstante, para suelos minerales de aptitud agropecuaria es limitada la vinculación entre suelos y su cubierta vegetal. Por consiguiente, los propósitos de este trabajo son establecer relaciones generales entre tres comunidades vegetales (murtillar, coironal y vegas) y las propiedades de los suelos minerales que las sustentan en Patagonia sur de Chile.

\section{MATERIALES Y MÉTODO}

Los sitios estudiados se localizan en comuna de Laguna Blanca, Provincia de Magallanes, Región de Magallanes y Antártica Chilena, $\sim 80 \mathrm{~km}$ al norte de la ciudad de Punta Arenas, por la Ruta 9, que la conecta con Puerto Natales. Climáticamente la zona corresponde a Subpolar Oceánica (Peel et al., 2007). En particular, el sector se incluye dentro del distrito agroclimático Puerto Natales-Punta Arenas, caracterizado por un verano fresco $\left(<16^{\circ} \mathrm{C}\right.$ como máxima del mes más cá- lido) y un invierno riguroso $\left(-0,5^{\circ} \mathrm{C}\right.$ de mínima media del mes más frío); además, presenta un período libre de heladas de solo 67 días, con 216 y 832 días-grado de acumulación de calor $\left(>10^{\circ} \mathrm{C} y>5^{\circ} \mathrm{C}\right.$, respectivamente), donde es posible desarrollar algunos cultivos anuales, hortalizas y praderas artificiales, quedando excluidos los frutales de clima templado y las especies exigentes en calor o sensibles a heladas (CNR, 1997).

La descripción de las comunidades vegetales en cada sitio (Cuadro 2) se realizó identificando sus especies dominantes y caracterizando su composición florística alrededor de la calicata empleada en la descripción morfológica de los suelos.

Conjuntamente con una descripción geomorfológica general de la zona que ocupan los sitios seleccionados para cada comunidad vegetal, se realizó la descripción de suelos, identificando horizontes genéticos en calicatas. En los horizontes más superficiales de cada calicata se tomaron muestras para realizar una caracterización básicamente química (Sadzawka et al., 2006). Se realizó la determinación de pH en agua y $\mathrm{CaCl}_{2}(1: 2,5)$, P-Olsen (extraído con bicarbonato de sodio, $\mathrm{pH} 8,5$ ), $\mathrm{S}$ disponible (extracción con $\mathrm{Ca}\left[\mathrm{H}_{2} \mathrm{PO}_{4}\right]_{2} 0,01 \mathrm{M}$ y determinación por turbidimetría), aluminio (Al) extractable (extraído con $\mathrm{CH}_{3} \mathrm{COONH}_{4} 1 \mathrm{M}, \mathrm{pH} 4,8$ y determinado por espectrofotometría de absorción atómica-EAA), cationes básicos (extraídos con $\mathrm{CH}_{3} \mathrm{COONH}_{4} 1 \mathrm{M}, \mathrm{pH} 7,0$ y determinación por EAA), $\mathrm{Al}$ de intercambio (extraído con $\mathrm{KCl} 1 \mathrm{M}$ y determinación por EAA), micronutrientes (extraídos con DTPA-CaCl 2 -TEA a pH 7,3 y determinación por EAA) y materia orgánica (digestión de Walkley-Black). La capacidad de intercambio catiónico efectiva (CICE), corresponde a la suma de cationes básicos y el Al intercambiable, en tanto que la saturación de $\mathrm{Al}$ se calcula como la proporción del Al de intercambio en la CICE (\%).

Cuadro 2. Caracterización general de los sitios evaluados y composición florística.

Table 2. General characterization of the sites evaluated and floristic composition.

\begin{tabular}{|c|c|c|c|c|}
\hline \multirow{2}{*}{ Sitios/comunidad vegetal } & \multirow{2}{*}{ Coordenadas (19F) } & Pendiente & Altitud & \multirow{2}{*}{ Especies vegetales asociadas } \\
\hline & & $\%$ & msnm & \\
\hline Murtillar & $350328 \mathrm{~S}-4170039 \mathrm{E}$ & $<1$ & 181 & $\begin{array}{l}\text { Empetrum rubrum, Festuca gracillima, Chillio- } \\
\text { trichum diffusum, Baccharis magellanica, Bolax } \\
\text { gummifera, Luzula alopecurusy Adesmia lotoides. }\end{array}$ \\
\hline Coironal & $348166 \mathrm{~S}-4176304 \mathrm{E}$ & $<1$ & 130 & $\begin{array}{l}\text { Festuca gracillima, C. diffusum, Azorella trifur- } \\
\text { cata, E. rubrum, Taraxacum officinale y L. alope- } \\
\text { curus. }\end{array}$ \\
\hline Vega & $350365 \mathrm{~S}-4170106 \mathrm{E}$ & 4 & 175 & $\begin{array}{l}\text { Poa sp., Trifolium repens, Juncus sp., Carex sp., } \\
\text { A. trifurcata, Leptinella scariosa; circundan el si- } \\
\text { tio, C. diffusum y Berberis microphylla. }\end{array}$ \\
\hline
\end{tabular}




\section{RESULTADOS Y DISCUSIÓN}

Se aprecia que los sitios se localizan en una zona de planicies y lomajes altos con algunos sectores ligeramente ondulados; este relieve obedece a una extensa red de drenaje de deshielos, que muestra una tendencia clara de dirección Oeste-Este, donde un complejo sistema de canales (sinuosos y anastomosados) submarginales y marginales se orientan al Este, cortando una gran llanura de depositación con alineaciones glaciales (ejemplo, drumlins). Esta descripción concuerda con las realizadas inicialmente por Clapperton (1989) y recientemente por Lovell et al. (2012). En la Figura 1 se incluyen los perfiles de suelo y su posición en el paisaje, asociados a cada comunidad vegetal estudiada.
De acuerdo a una síntesis de la descripción morfológica de cada uno de los horizontes genéticos identificados (Cuadro 3), en general, los pedones de los sitios de coironal y murtillar, cumplen con las características descritas para la serie de suelos Última Esperanza (CNR, 1997). Se trata de suelos delgados, formados por sedimentos fluvioglaciales depositados sobre materiales glaciolacustres; se presentan compactados y extraordinariamente duros, no cementados y de texturas finas, determinando que las raíces sólo penetren por las fracturas que se generan. Se ubican en las zonas más altas (pampas), presentando buen drenaje.

El suelo de vega, se podría asociar a la Serie Tehuelche (CNR, 1997) formada por sedimentos lacustrinos finos, pero en general pueden encontrarse por toda la

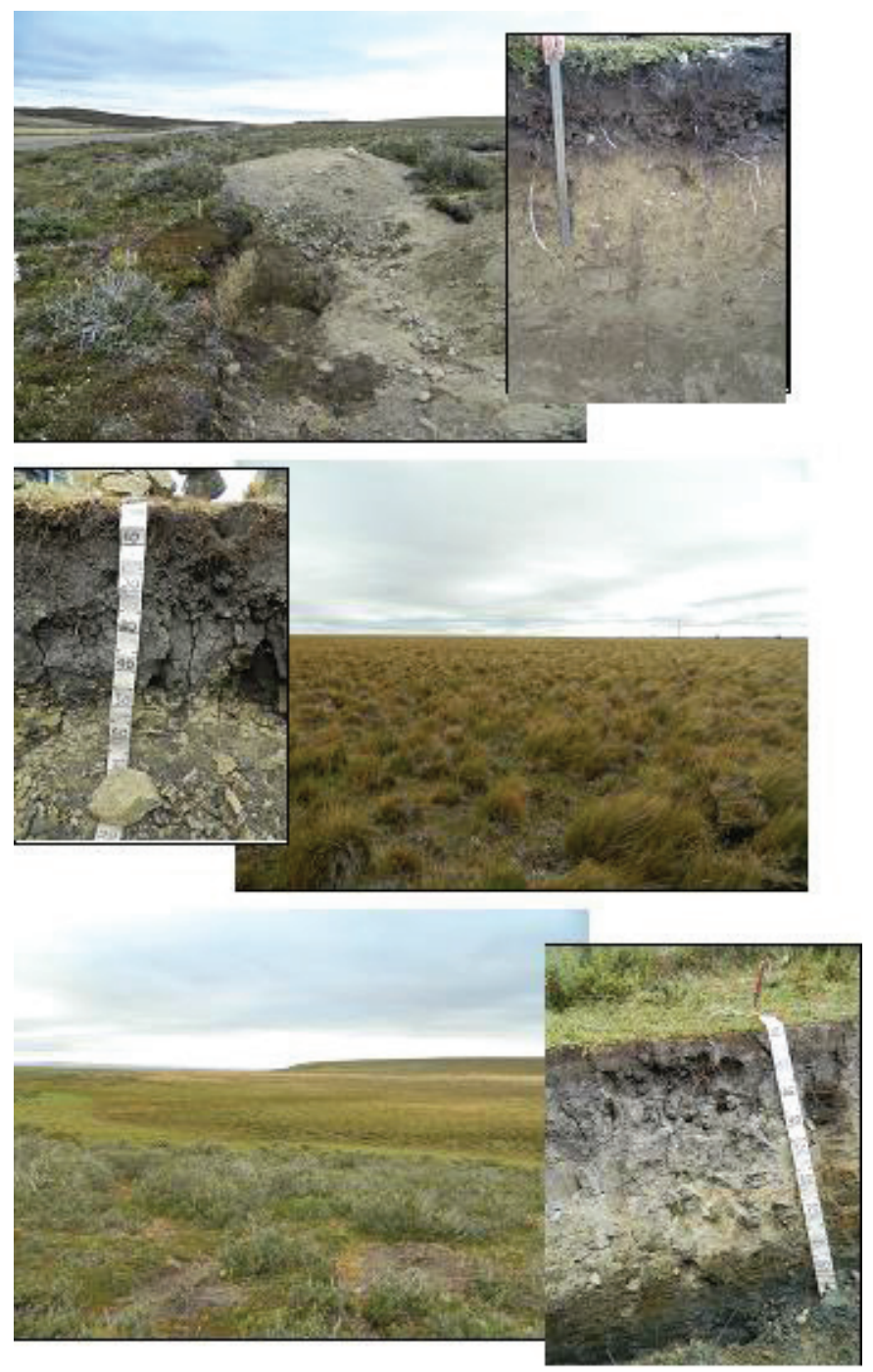

Figura 1. Pedones descritos, su posición en el paisaje y vegetación natural residente. Suelo bajo murtilla (superior), coirón (medio) y vega (inferior), en Patagonia Sur.

Figure 1. Described pedons, its position on landscape and natural resident vegetation. Soil under murtilla (upper), coirón (middle) and vega (lower) in South Patagonia. 
Cuadro 3. Resumen de la descripción morfológica de los suelos.

Table 3. Summary of the morphology soil description.

\begin{tabular}{|c|c|c|c|c|c|c|c|c|c|}
\hline Sitio & $\mathrm{HG}$ & Prof. (cm) & Color & Clase textural & Estructura & Consistencia & Raíces & Poros & Límite \\
\hline \multirow{4}{*}{ Murtillar } & $\mathrm{A} 1$ & $0-20$ & 10 YR $2 / 1$ & $\mathrm{Fa}$ & G gr mo & IP y nA & $\begin{array}{c}\text { Fy M ab } \\
\text { G co }\end{array}$ & G ab, FM co & $\mathrm{LC}$ \\
\hline & $\mathrm{A} 2$ & $20-32$ & 10 YR $3 / 2$ & $\mathrm{~F}$ & G mgr mo & P y lA & $\begin{array}{c}\text { F y M ab } \\
\text { G co }\end{array}$ & GMF ab & $\mathrm{OA}$ \\
\hline & $\mathrm{Bw}$ & $32-78$ & 10 YR 6/4 & $\mathrm{FA}$ & $\mathrm{BS}$ me $\rightarrow$ gr mo & Py A & M y G es & G es, F ab & OC \\
\hline & $2 \mathrm{C}$ & $78-100$ & 2.5 Y $6 / 4$ & FAL & L gr mo & Py A & - & $\mathrm{Fab}$ & - \\
\hline \multirow{5}{*}{ Coironal } & $\mathrm{Oe}$ & $0-6$ & 10 YR $2 / 1$ & - & - & - & Fy M ab & & LC \\
\hline & $\mathrm{A} 1$ & $6-20$ & 2.5 Y $2.5 / 1$ & FL & G me f & IP y lA & F y M co & G es, Mco, F ab & OC \\
\hline & $\mathrm{A} 2$ & $20-35$ & 2.5 Y $2.5 / 1$ & FA & P gr f & Py A & F es & M co, F ab & OG \\
\hline & B & $35-50^{* *}$ & 2.5 Y $3 / 2$ & A & Pgr f & Py mA & - & G co, MF ab & OC \\
\hline & $2 \mathrm{Cg}$ & $50-64^{*}$ & 2.5 Y $6 / 4$ & A & L gr mo & Py mA & - & MF ab & - \\
\hline \multirow{5}{*}{ Vega } & Oe & $0-9$ & 5 YR 3/3 & - & - & - & F y M ab & & LC \\
\hline & $\mathrm{Oa}$ & $9-23$ & 5 YR 3/1 & - & - & - & F y M ab & & $\mathrm{OA}$ \\
\hline & A & $23-36$ & 5 YR 3/1 & A & G mgr de & Py mA & Fy M es & $\mathrm{F} \mathrm{ab}$ & OC \\
\hline & $2 \operatorname{Cg} 1$ & $36-70$ & 5 YR 4/1 & A & M & Py mA & F es & MF F ab & $\mathrm{OA}$ \\
\hline & $2 \mathrm{Cg} 2$ & $70-90$ & Gley $14 / 1$ & A & M & $\mathrm{mPy} \mathrm{mA}$ & - & $\mathrm{MF}$ ab & - \\
\hline
\end{tabular}

HG: horizonte genético. Clase textural: franca (F), arcillosa (A), limosa (L), arenosa (a). Estructura: bloques subangulares (BS), laminar (L), granular (G), prismática (P), masiva (M); medios (me), gruesos (gr), muy gruesos (mgr); fuerte (f), moderada (mo) y débil (de). Consistencia: plástica (P), adhesiva (A), muy (m) ligeramente (l). Raíces: finas (F), medias (M), gruesas (G); escasas (es), comunes (co), abundantes (ab). Poros: gruesos (G), medios (M), finos (F), muy finos (MF), muy abundantes (mab), abundantes (ab), comunes (co), escasos (es). Límite: lineal $(\mathrm{L})$, ondulado $(\mathrm{O})$; claro $(\mathrm{C})$, gradual $(\mathrm{G})$ y abrupto $(\mathrm{A}) .\left(^{* *}\right)$ presencia de organes abundantes y $\left({ }^{*}\right)$ comunes.

Región. Filipová et al. (2010) los clasifican como Histosoles y Fluvisoles, informando que existe una considerable variabilidad en su contenido de materia orgánica (MO) y $\mathrm{pH}$, tendiendo a salinizarse en condiciones de aridez y riesgos de deterioro cuando son drenados. Si bien la pradera que presentan suele presentar mayor productividad, sólo pueden usarse en el período estival por encontrarse inundados gran parte del año. Los análisis de este estudio indican que este suelo se podría clasificar como Humaquept.

Normalmente se distinguen diferentes tipos de coirón (Festuca pallescens, Stipa speciosa o S. milis y F. gracillima) cuya distribución correspondería a diferencias de suelos o condiciones climáticas (IREN, 1967). Así, el suelo bajo coirón estudiado en este trabajo posee un epipedón móllico (Humoll) y desde los $35 \mathrm{~cm}$ en profundidad presenta abundante presencia de cutanes de MO (organes), a diferencia del suelo bajo murtillar cuyo horizonte superficial presenta una saturación básica < 50 \% y materiales sápricos (Humudept).

Para el suelo bajo el murtillar se observa una cobertura de vegetación discontinua, la presencia de pedestales de plantas y abundantes fragmentos gruesos superficia- les, indicadores de una erosión eólica severa. Morfológicamente, no se diferencian los horizontes orgánicos superficiales, lo que confirma la presencia de este selectivo proceso degradativo. Collantes et al. (1989) señalan que la pérdida de suelos puede alcanzar un equivalente a 30 $40 \mathrm{~cm}$ de los horizontes superficiales, en donde el subsuelo gravoso queda expuesto. Borrelli y Oliva (2001) observan este mismo rasgo en el murtillar, en tanto que Mendoza et al. (2011) atribuyen estos procesos a un pastoreo selectivo prolongado de elevada intensidad.

Collantes et al. (1999) señalan que los suelos bajo murtilla (principalmente Tierra del Fuego) se caracterizan por una textura gruesa, ácidos y pobres en nutrientes, con MO poco descompuesta, bajos en cationes no ácidos y alta relación $\mathrm{C} / \mathrm{N}$, lo cual obedece más bien a una elevada saturación de $\mathrm{Al}$ e hidrólisis de éste en los suelos, que a la pérdida de cationes no ácidos ( $\mathrm{Ca}, \mathrm{Mg}$, Na y K). En cambio, los suelos bajo coirón y vega, muestran (Figura 2) una tendencia a presentar una suma de cationes no ácidos alta a muy alta y una saturación básica cercana a 100\% (Bernier, 2000); así, es posible inferir que existe transferencia de estos elementos, al menos entre murtillar y vega. 


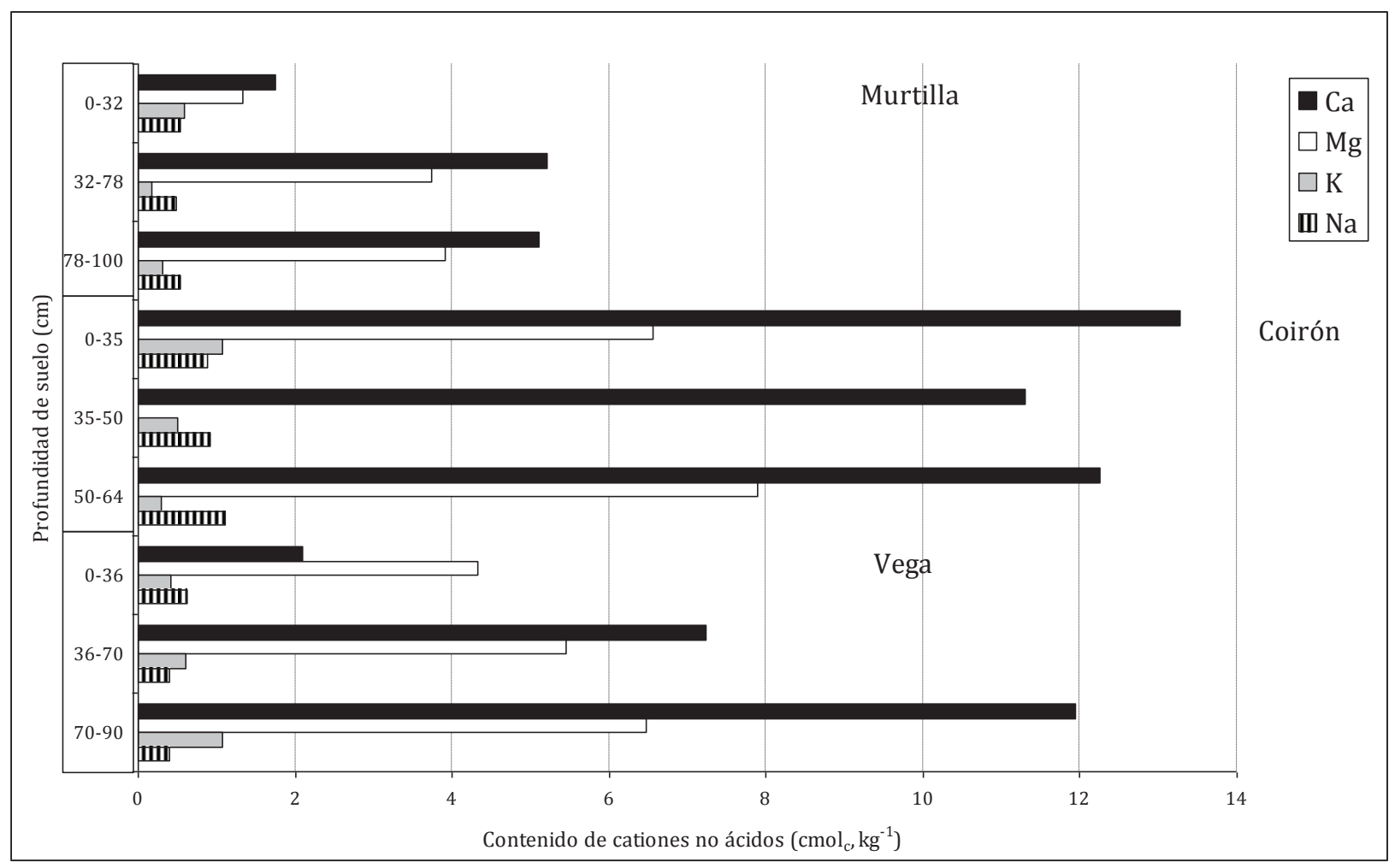

Figura 2. Cationes no ácidos en profundidad de los suelos, en las distintas comunidades vegetales analizadas. Patagonia Sur.

Figure 2. Non acid cations in soil depth under the three different analyzed vegetal communities. South Patagonia.

Los rangos deseables (Jones, 2012) de estos cationes en el complejo de intercambio son $60 \%$ a $80 \%$ (Ca), $15 \%$ a $20 \%$ (Mg) y $5 \%$ a $10 \%(\mathrm{~K})$. Salvo para el murtillar en superficie, en los suelos estudiados, solo Mg se encuentra en niveles satisfactorios; para Ca, solo en el caso del coironal; para $\mathrm{K}$, son satisfactorios o cercanos a este valor en todo el suelo bajo vega, y solo en superficie de los otros dos suelos. De acuerdo a la relación $\mathrm{K} / \mathrm{Mg}$, todos los suelos en superficie muestran niveles deficientes de $\mathrm{Mg}$ por su antagonismo con $\mathrm{K}$, pero el suelo bajo vega los muestra en todo el perfil (Bernier, 2000). George et al. (2012), informan a diferencia de Ca y Mg, en general la absorción de K no se ve afectada negativamente por el $\mathrm{Al}$, lo que lleva a un aumento en la relación $\mathrm{K} / \mathrm{Ca}+\mathrm{Mg}$ en la fracción aérea de las plantas, aumentando el riesgo de deficiencia de Ca o Mg en ellas, así como de hipomagnesemia en rumiantes.

Se puede apreciar (Figura 3) que, ya sea medido en agua o $\mathrm{CaCl}_{2}$, efectivamente el suelo bajo murtillar posee los valores de $\mathrm{pH}$ más bajos de los 3 sitios. La toxicidad por Al es considerada un factor limitante primario del crecimiento de plantas en suelos fuertemente ácidos (Fageria et al., 2011). En la medida que el pH del suelo decrece, el contenido de Al se incrementa (Figura 4), lo que también es observado por Hart y Mellbye (2010) destacando una reducción linear de la producción de semillas en praderas. A este hecho, se agrega una elevada saturación de $\mathrm{Al}$ o un exceso de $\mathrm{Al}$ en la solución del suelo, que interfiere en la división celular y reduciría el crecimiento de raíces (Bernier, 2000). Campillo y Sadzawka (2006) precisan que el principal efecto de la toxicidad por Al es la restricción del desarrollo radicular, que reduce el volumen de suelo a explorar y la eficiencia en la absorción tanto de nutrientes como de agua; además, interfiere en el transporte y utilización de nutrientes esenciales e inhibe los procesos microbianos que suministran nutrientes a las plantas.

El comportamiento de $\mathrm{Al}$ en suelos es altamente modificado por la MO, debido a las formas de Al unidas orgánicamente tanto en fase sólida como líquida; en este estudio se obtiene una relación lineal de los contenidos de $\mathrm{MO}$ con el Al intercambiable $\left(\mathrm{R}^{2}: 0,43\right)$, el Al extraíble $\left(\mathrm{R}^{2}: 0,53\right)$ y la saturación de $\mathrm{Al}\left(\mathrm{R}^{2}: 0,46\right)$.

En cuanto a micronutrientes (Figura 5) para todos los suelos los niveles de $\mathrm{Cu}$ son bajos, los niveles de Mn son altos a muy altos solo en el caso del suelo bajo vega, el $\mathrm{Fe}$ tiende a ser alto a muy alto en la superficie de todos los suelos y el Zn solo presenta valores altos en los dos primeros horizontes del suelo del coironal (Jones, 2003).

Para todos los sitios (Cuadro 4) los niveles de S registrados son bajos y normales para suelos del sur de Chile, lo que afecta la calidad del forraje, pues su esca- 


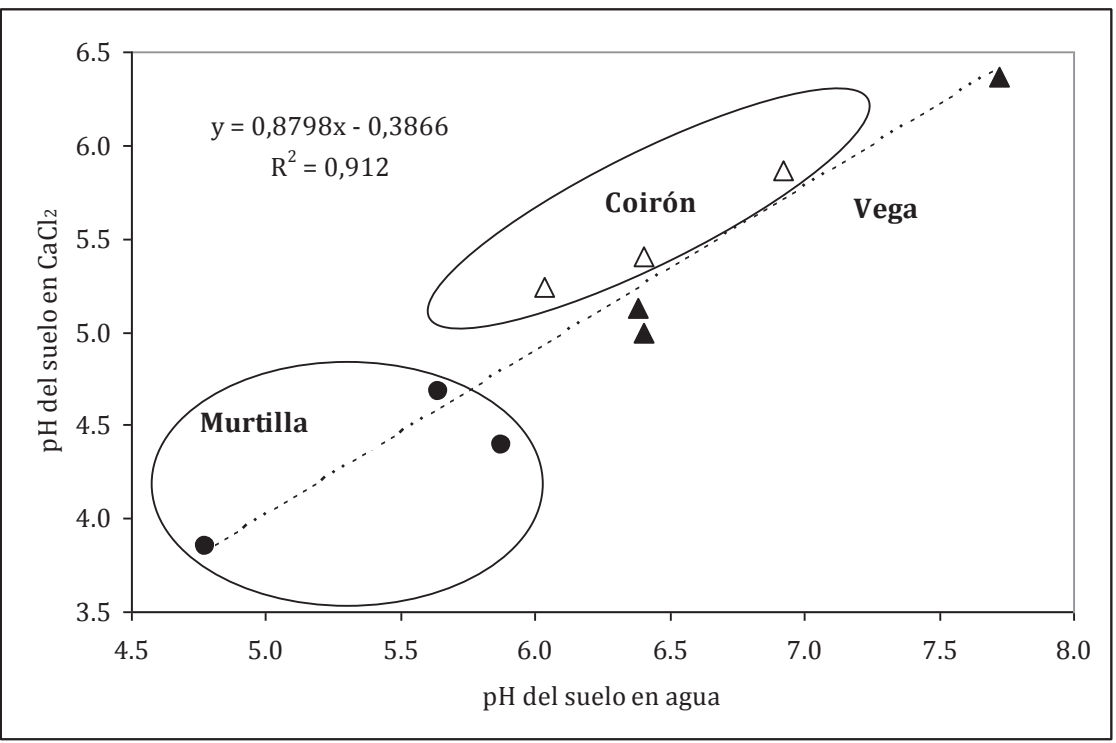

Figura 3. Valores de $\mathrm{pH}$ en agua y $\mathrm{CaCl}_{2}$, medido en suelos bajo tres comunidades vegetales. Patagonia Sur.

Figure 3. Soil $\mathrm{pH}$ in water and $\mathrm{CaCl}_{2}$ measured under the three vegetal communities. South Patagonia.

Cuadro 4. Característica química de los suelos evaluados bajo tres comunidades vegetales en Patagonia Sur.

Table 4. Chemical soil properties under three vegetal communities in South Patagonia.

\begin{tabular}{|c|c|c|c|c|c|c|c|c|c|c|}
\hline & \multirow{2}{*}{$\frac{\text { Sitio }}{\text { Unidades }}$} & \multicolumn{3}{|c|}{ Murtillar } & \multicolumn{3}{|c|}{ Coironal } & \multicolumn{3}{|c|}{ Vega } \\
\hline & & A1-A2 & $\mathrm{Bw}$ & $2 \mathrm{C}$ & Oe-A1-A2 & $\mathrm{B}$ & $2 \operatorname{Cg} 1$ & Oe-Oa-A & 2Cg1 & 2Cg2 \\
\hline Prof. & $\mathrm{cm}$ & $0-32$ & $32-78$ & $78-100$ & $0-35$ & $35-50$ & $50-64$ & $0-36$ & $36-70$ & $70-90$ \\
\hline $\mathrm{pH}-\mathrm{H}_{2} \mathrm{O}$ & - & 4,78 & 5,88 & 6,40 & 6,03 & 6,40 & 6,92 & 5,64 & 6,38 & 7,72 \\
\hline $\mathrm{pH}-\mathrm{CaCl}_{2}$ & - & 3,85 & 4,39 & 4,99 & 5,24 & 5,40 & 5,87 & 4,68 & 5,13 & 6,37 \\
\hline P-Olsen & \multirow{7}{*}{$\mathrm{mg} \mathrm{kg}^{-1}$} & 6,09 & 3,32 & 13,67 & 4,51 & 1,37 & 1,48 & 4,75 & 41,15 & 3,46 \\
\hline Azufre & & 6,87 & 0,24 & 0,22 & 0,26 & 0,22 & 0,20 & 0,26 & 0,19 & 0,13 \\
\hline Al extr & & 748 & 174 & 57 & 22 & 30 & 28 & 110 & 25 & 20 \\
\hline $\mathrm{Zn}_{\mathrm{DTPA}}$ & & 1,16 & 0,44 & 0,46 & 4,05 & 1,36 & 0,66 & 0,99 & 0,79 & 0,43 \\
\hline $\mathrm{Fe}_{\text {DTPA }}$ & & 424,00 & 3,65 & 3,06 & 304,13 & 220,13 & 2,96 & 240,40 & 6,74 & 1,62 \\
\hline $\mathrm{Mn}_{\mathrm{DTPA}}$ & & 0,55 & 1,89 & 2,52 & 7,49 & 3,11 & 6,74 & 22,58 & 23,40 & 260,75 \\
\hline $\mathrm{Cu}_{\mathrm{DTPA}}$ & & 1,16 & 0,71 & 0,62 & 1,51 & 1,96 & 1,67 & 1,97 & 1,59 & 3,26 \\
\hline $\mathrm{Ca}$ & \multirow{6}{*}{$\mathrm{cmol}_{\mathrm{c}} \mathrm{kg}^{-1}$} & 1,75 & 5,21 & 5,10 & 13,27 & 11,30 & 12,25 & 2,09 & 7,24 & 11,94 \\
\hline $\mathrm{Mg}$ & & 1,33 & 3,74 & 3,91 & 6,56 & 6,07 & 7,89 & 4,33 & 5,45 & 6,47 \\
\hline $\mathrm{K}$ & & 0,58 & 0,17 & 0,31 & 1,07 & 0,50 & 0,29 & 0,41 & 0,61 & 1,08 \\
\hline $\mathrm{Na}$ & & 0,54 & 0,48 & 0,54 & 0,88 & 0,92 & 1,10 & 0,63 & 0,39 & 0,40 \\
\hline Al int. & & 5,23 & 1,50 & 0,26 & 0,01 & 0,01 & 0,01 & 0,12 & 0,13 & 0,01 \\
\hline CICE & & 9,44 & 11,10 & 10,12 & 21,78 & 18,80 & 21,54 & 7,58 & 13,81 & 19,91 \\
\hline Sat. Al & \multirow{3}{*}{$\%$} & 55,44 & 13,50 & 2,62 & 0,04 & 0,07 & 0,05 & 1,54 & 0,91 & 0,06 \\
\hline MO & & 30,75 & 4,74 & 3,69 & 19,45 & 9,39 & 5,61 & 20,87 & 4,03 & 3,88 \\
\hline SB & & 44,5 & 86,5 & 97,4 & 100 & 99,9 & 100 & 98,4 & 99,1 & 99,9 \\
\hline Suma de cationes & $\mathrm{cmol}_{\mathrm{c}} \mathrm{kg}^{-1}$ & 4,20 & 9,60 & 9,86 & 21,78 & 12,72 & 21,53 & 7,46 & 13,69 & 19,89 \\
\hline
\end{tabular}

Al extr: aluminio extraíble; Al int: aluminio intercambiable; Sat Al: saturación de aluminio; MO: materia orgánica; CICE: capacidad de intercambio efectiva; SB: saturación básica o de cationes no ácidos. 

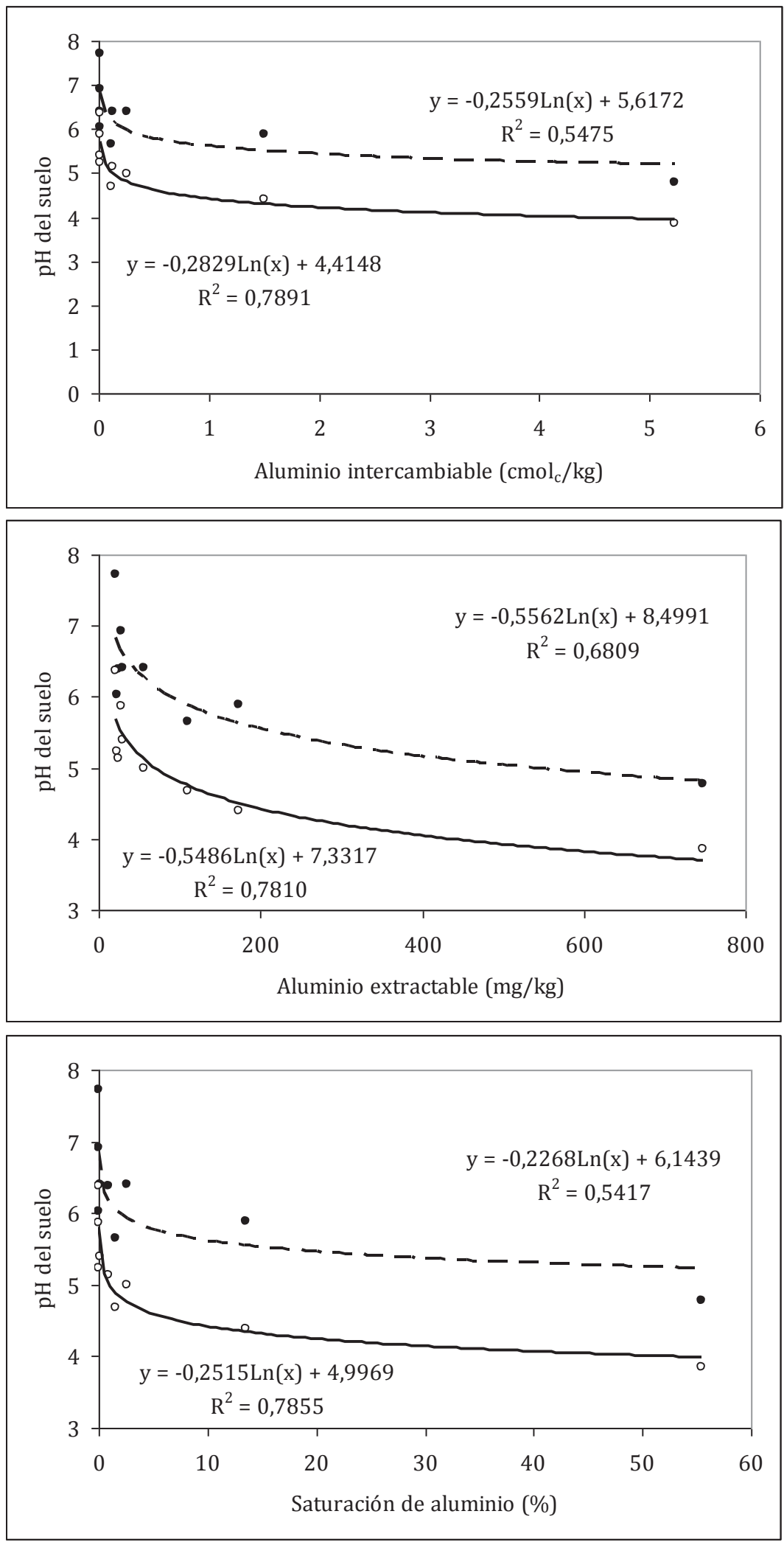

Figura 4. Relación entre el pH de suelos (medido en agua, círculos negros; en $\mathrm{CaCl}_{2}$, círculos blancos), el contenido (extractable e intercambiable) y la saturación de aluminio en Patagonia Sur.

Figure 4. Relationships between soil $\mathrm{pH}$ (measured in water, black circles; in $\mathrm{CaCl}_{2}$, white circles), saturation and aluminum content (extractable and exchangeable) in South Patagonia. 

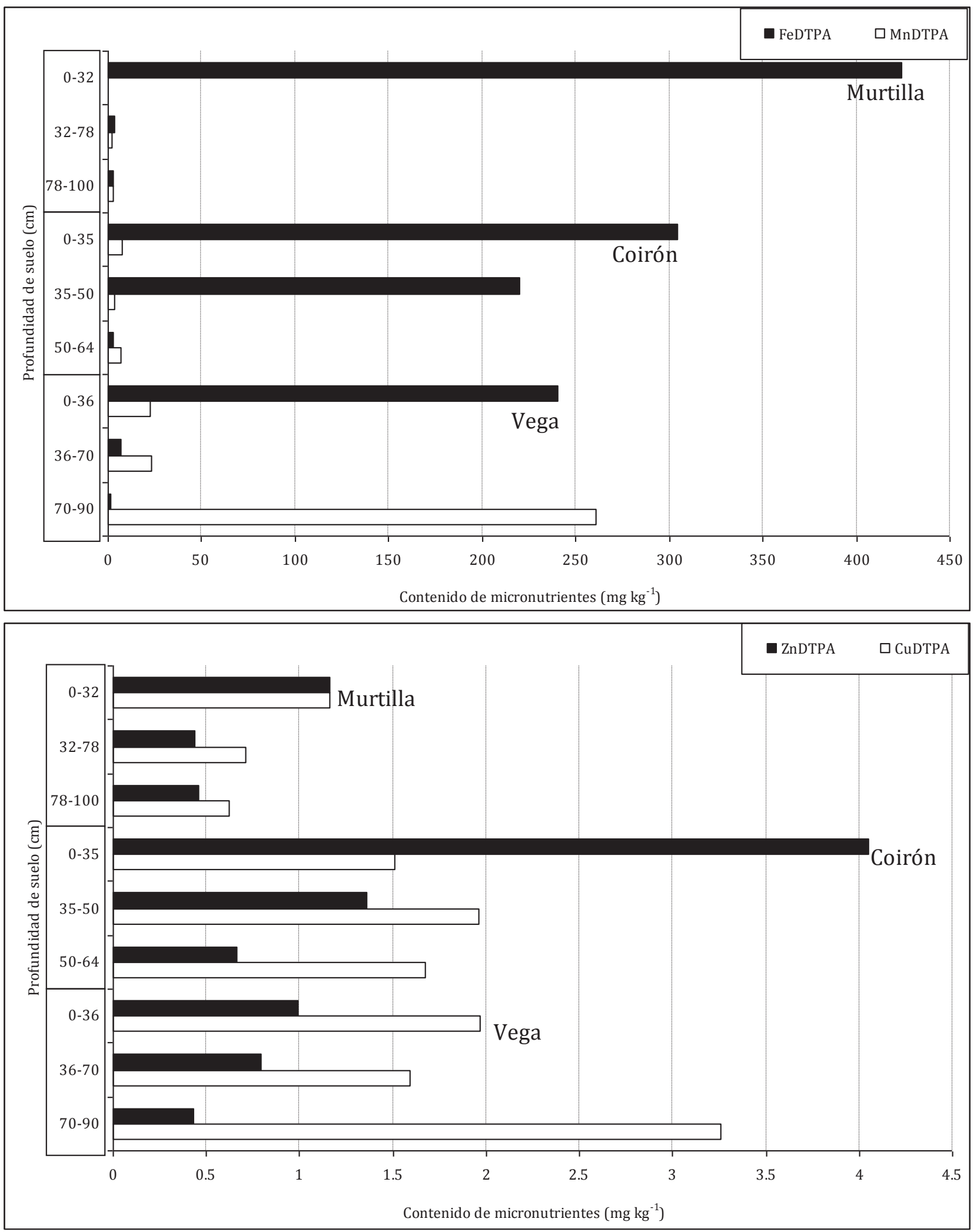

Figura 5. Contenido de micronutrientes en los suelos de las comunidades vegetales estudiadas. Patagonia Sur.

Figure 5. Micronutrient content of soils in each vegetal community. South Patagonia. 
sez acelera la pérdida de digestibilidad y conlleva un menor potencial de transformación en leche o carne. Los niveles de P son en general bajos a muy bajos (Bernier, 2000; Ponce, 2000).

\section{CONCLUSIONES}

La zona definida como Patagonia Sur (al sur del paralelo $53^{\circ} \mathrm{S}$ ) cuenta con registros de clima y suelos suficientes, aunque de estos últimos la información es dispersa y poco sistematizada. En el área con precipitaciones entre $350-450 \mathrm{~mm}$, sobre un paisaje fluvioglacial, existe una clara diferenciación de comunidades arbustivo-esteparias asociada al tipo de suelo.

Los perfiles de estos suelos se diferencian claramente en términos morfológicos, además de presentar propiedades físicas, química y fisicoquímicas diferentes.

En general, se verifica un gradiente de fertilidad de los suelos. Los suelos bajo murtilla, en posiciones topográficas más elevadas, presentan una menor fertilidad, asociada a una restricción impuesta por un pH bajo, altos niveles de aluminio y bajos niveles de cationes no ácidos en el suelo superficial. Con un valor forrajero bajo, la presencia de E. rubrum implica que esos sectores pueden utilizarse con bajas cargas animales.

La comunidad de vega presenta suelos de mayor hidromorfismo, con periodos prolongados de saturación, lo que se evidencia en los rasgos redoximórficos y de gleización del perfil. Esto se explica por su posición en depresiones del relieve, concentrándose en ellas además de agua, sedimentos y sales de los sectores circundantes, favoreciéndola con una mejor fertilidad, comparada con el murtillar.

Las características del perfil estudiado bajo la comunidad de coirón (alta suma de cationes no ácidos, $\mathrm{pH}$ moderada a débilmente ácido, bajo contenido de $\mathrm{Al}$ y ausencia de hidromorfismo) indican que han experimentado procesos de menor intensidad que los anteriores. Su menor elevación y exposición a menores precipitaciones probablemente los ha favorecido.

\section{AGRADECIMIENTOS}

Los autores agradecen la valiosa cooperación del Dr. James Bockheim (University of Wisconsin-Madison) y al Dr. Jorge Irisarri (Universidad de Comahue).

\section{REFERENCIAS}

Anchorena, J., Cingolani, A.M., Livraghi. E, Collantes, M., Stoffella, S., 2001. Manejo del pastoreo de ovejas en Tierra del Fuego. EDIPUBLI SA, Buenos Aires.

Bernier, R., 2000. Diagnóstico de la fertilidad de suelos, in: Bernier, R., Bortolameolli, G. (Eds.), Técnicas de diagnóstico de fertilidad del suelo, fertilización de praderas, cultivos y mejoramiento de praderas. Instituto de Inves- tigaciones Agropecuarias-Centro Regional de Investigación Remehue, Serie Actas № 4. Osorno, Chile. pp. 1-15.

Borrelli, P., Oliva, G., 2001. Efecto de los animales sobre los pastizales, in: Borrelli, P., Oliva, G. (Eds.), Ganadería ovina sustentable en la Patagonia Austral. Estación Experimental Agropecuaria de Santa Cruz. Instituto Nacional de Tecnología Agropecuaria (INTA). Buenos Aires, Argentina. pp. 99-128.

Campillo, R., Sadzawka, A., 2006. La acidificación de los suelos. Origen y mecanismos involucrados, in: Campillo, R. (Ed.). Manejo de los recursos naturales en el Sistema de Incentivos para la Recuperación de Suelos Degradados de la Araucanía. Instituto de Investigaciones Agropecuarias-Centro Regional de Investigación Carillanca, Temuco, Chile. Serie Actas $N^{\circ} 38$. pp. 44-60.

Centro de Información en Recursos Naturales (CIREN). 2010. Determinación de la erosión actual y potencial de los suelos de Chile. Región de Magallanes y Antártica Chilena. Síntesis de resultados. Santiago, Chile.

Cibils, A.F., Borrelli P.R., 2005. Grasslands of Patagonia. in: Suttie, J.M., Reynolds, S.G., Batello, C. (Eds.). Grasslands of the World. FAO, Rome. pp. 121-170.

Clapperton, C.M., 1989. Asymmetrical drumlins in Patagonia, Chile. Sedimentary Geology 62, 387-398. doi: 10.1016/0037-0738(89)90127-9

Collantes, M.B., Anchorena, J., Koremblit, G., 1989. A soil nutrient gradient in Magellanic Empetrum heathlands. Vegetatio 80, 183-193. doi: 10.1007/BF00048041

Comisión Nacional de Riego (CNR), 1997. Estudio Integral de Riego y Drenaje de Magallanes, XII Región. Volumen 2. Introducción, aspectos metodológicos, caracterización general del área y estudios básicos. Punta Arenas, Chile.

Collantes, M., Anchorena, J., Cingolani, A., 1999. The steppes of Tierra del Fuego: floristic and growth form patterns controlled by soil fertility and moisture. Plant Ecology 140, 61-75. doi: 10.1111/j.1365-2664.2004.00978.x

Cruz, G., Lara, A., 1987. Vegetación del área de uso agropecuario de la XII Región de Magallanes y la Antártica Chilena. Instituto Nacional de Investigaciones Agropecuarias, Santiago, Chile.

Dekker, S.C., de Boer, H.J., Brovkin, V., Fraedrich, K., Wassen, M.J., Rietkerk, M., 2010. Biogeophysical feedbacks trigger shifts in the modelled vegetation-atmosphere system at multiple scales. Biogeosciences 7, 1237-1245. doi: 10.5194/bg-7-1237-2010

Díaz, C., Avilés, C., Roberts, R., 1959-1960. Los grandes suelos de la Provincia de Magallanes. Agricultura Técnica 19/20, 227-308.

Fageria, N.K., Baligar, V.C., Jones, C.A., 2011. Growth and Mineral Nutrition of Field Crops. $3^{\text {th }}$ Edition, CRC Taylor \& Francis Group. Boca Raton, USA, Florida.

Filipová, L., Héld, R., Covacevich, N., 2010. Variability of soil types in wetland meadows in the south of the Chilean Patagonia. Chilean Journal of Agricultural Research 70(2), 266-277. doi: 10.4067/S0718-58392010000200010

Frederiksen, P., 1988. Soils of Tierra del Fuego. A satellitebased land survey approach. Folia Geographica Danica 18, 1-159.

George, E., Horst, W., Neumann, E., 2012. Adaptation of Plants to Adverse Chemical Soil Conditions, in: Marschner P. (Ed.), Marschner`s Mineral Nutrition of Higher 
Plants, Third Edition. Elsevier/Academic Press, London; Waltham, MA. pp. 409-472.

Gerding, V., Thiers, O., 2002. Caracterización de suelos bajo bosques de Nothofagus betuloides (Mirb) Blume, en Tierra del Fuego, Chile. Revista Chilena de Historia Natural 75, 819-833. doi: 10.4067/S0716-02082003000100002

Hart, J.M., Mellbye, M.E., 2010. Annual ryegrass seed production in acidic soil, in: Young III, W.C. (Ed.), Seed production research at Oregon State University, Department of Crop and Soil Science, Ext/CrS, 129. pp. 9-12.

Instituto de Investigación de Recursos Naturales (IREN), 1967. Provincia de Magallanes. Inventario preliminar de los recursos naturales. Zona continental e Isla Tierra del Fuego. Instituto de Investigación de Recursos Naturales. Informe $\mathrm{N}^{\circ} 21$. Santiago, Chile.

Instituto de Investigación de Recursos Naturales (IREN), 1968. Asociaciones de Suelos de la Provincia de Magallanes (Zona Continental). Informe $\mathrm{N}^{\circ} 24$. Santiago, Chile.

Isla, F.I., Bujalesky, G., 2008. Coastal Geology and morphology of Patagonia and Fueguian Archipielago, in: Rabassa J.R. (Ed.), The Late Cenozoic of Patagonia and Tierra del Fuego. Elsevier Science Publishers 10. pp. 227-240.

Jones, B., 2003. Agronomic Handbook: Management of Crops, Soils, and their Fertility. CRC Press. Boca Raton, USA.

Jones, B., 2012. Plant Nutrition and Soil Fertility Manual. $2^{\text {nd }}$ Ed. CRC Press. Boca Raton, USA.

Loisel, J., Yu, Z., 2013. Holocene peatland carbon dynamics in Patagonia. Quaternary Science Reviews 69, 125-141. doi: 10.1016/j.quascirev.2013.02.023

Lovell, H. Chris, R., Stokes, M., Bentley, J., Benn, D.I., 2012. Evidence for rapid ice flow and proglacial lake evolution around the central Strait of Magellan region, southernmost Patagonia. Journal of Quaternary Sciences 27(6), 625-638. doi: 10.1002/jqs.2555

Maestre, F. T., Cortina, J., 2002. Spatial patterns of surface soil properties and vegetation in a Mediterranean semi-arid steppe. Plant and Soil 241, 279-291. doi: 10.1023/A:1016172308462

McGrath, G., Paik, K., Hinz C., 2012. Microtopography alters self-organized vegetation patterns in water-limited ecosystems. Journal of Geophysical Research 117, G03021, doi:10.1029/2011JG001870

Mendoza, R., Cabello, M., Anchorena, J., García, I., Marbán, L., 2011. Soil parameters and host plants associated with arbuscular mycorrhizas in the grazed Magellanic steppe of Tierra del Fuego. Agriculture, Ecosystem and Environment 140, 411-418. doi: 10.1016/j.agee.2011.01.004

Paruelo, J.M., Golluscio, R.A., Jobbágy, E.G., Canevari, M., Aguiar, M.R., 2006. Situación ambiental en la estepa Patagónica, In: A. Brown, U. Martinez Ortiz, M. Acerbi \& J. Corchera (eds), La Situación Ambiental Argentina 2005. Fundación Vida Silvestre, Buenos Aires, Argentina. pp. 302-320.

Peel, M.C., Finlayson, B.L., Mcmahon, T.A., 2007. Updated world map of the Köppen-Geiger climate classification. Hydrology and Earth System Sciences 11, 1633-1644. doi: 10.5194/hess-11-1633-2007

Pisano, E., 1973. Fitogeografía de la Península de Brunswick, Magallanes. Comunidades meso-higromórficas e higromórficas. Anales Instituto Patagonia (Chile) 4, 141-206.

Pisano, E., 1974. Estudio ecológico de la región continental sur del área andino-patagónica. II. Contribuciones a la fitogeografía de la zona de, P.N. Torres del Paine. Anales Instituto Patagonia (Chile) 5, 59-104.

Pisano, E., 1977. Fitogeografía de Fuego-Patagonia Chilena. I.- Comunidades vegetales entre las latitudes 52 y $56^{\circ}$. Anales Instituto Patagonia (Chile) 8, 121-250.

Pisano, E., 1993. Biogeografía. Instituto de la Patagonia, Universidad de Magallanes. Punta Arenas. Chile.

Ponce, M., 2000. Aspectos económicos de la fertilización de praderas y cultivos, in: Bernier, R., Bortolameolli, G. (Eds.), Técnicas de diagnóstico de fertilidad del suelo, fertilización de praderas, cultivos y mejoramiento de praderas. Instituto de Investigaciones AgropecuariasCentro Regional de Investigación Remehue, Serie Actas $\mathrm{N}^{\circ}$ 4. Osorno, Chile. pp. 58-70.

Ramírez, C., Sandoval, V., San Martín, C., Álvarez, M., Pérez, Y. y Novoa, C., 2012. El paisaje rural antropogénico de Aisén: Estructura y dinámica de la vegetación. Gayana Botanica, 69(2), 219-231. doi: 10.4067/S071766432012000200001

Rietkerk, M., Boerlijst, M.C., van Langevelde, F., Hille Ris Lambers, R., van de Koppel, J., Kumar, L., Prins, H.H.T., de Roos, A.M., 2002. Self-organization of vegetation in arid ecosystems. The American Naturalist 160(4), 524-530. doi: $10.1086 / 342078$

Rodríguez, S.O., 1986. Sistemas de Producción, in: Estudio para un Plan de Desarrollo tecnológico agropecuario en la XII Región. 2a Etapa, Instituto de Investigaciones Agropecuarias- Secretaría Regional de Planificación y Coordinación. Región de Magallanes. pp. 67-72.

Roig, F., 1998. La vegetación de la Patagonia. in: Correa M. (Ed.), Flora Patagónica Parte I. pp. 48-116. Colección Científica INTA. Buenos Aires, Argentina.

Romanya, J., Fons, J., Sauras-Yera, T., Gutierrez, E., Vallejos, V., 2005. Soil-plant relationships and tree distribution in old growth Nothofagus betuloides and Nothofagus pumilio forests of Tierra del Fuego. Geoderma 124, 169-180. doi:10.1016/j.geoderma.2004.04.011

Sadzawka, A., Carrasco, M.A., Grez, R., Mora, M.L., Flores, H., Neaman, A., 2006. Métodos de Análisis Recomendados para Suelos de Chile. Comisión de Normalización y Acreditación (CNA). Serie Actas INIA N³4. Santiago, Chile.

Servicio Agrícola y Ganadero (SAG), 2003. El pastizal de Tierra del Fuego. Guía de uso, condición actual y propuesta de seguimiento para determinación de tendencia. Gobierno de Chile. Punta Arenas, XII Región de Magallanes y Antártica Chilena, Chile.

Servicio Agrícola y Ganadero (SAG), 2004a. El pastizal de Magallanes. Guía de uso, condición actual y propuesta de seguimiento para determinación de tendencia. Gobierno de Chile. XII Región de Magallanes y Antártica Chilena, Punta Arenas, Chile.

Servicio Agrícola y Ganadero (SAG), 2004b. El pastizal de Última Esperanza y Navarino. Guía de uso, condición actual y propuesta de seguimiento para determinación de tendencia. Gobierno de Chile. XII Región de Magallanes y Antártica Chilena, Punta Arenas, Chile.

Zaixso, H., Boraso, A., 2015. La zona costera patagónica argentina: comunidades biológicas y geología. Universitaria de la Patagonia-EDUPA. Comodoro Rivadavia, Argentina. 
